\title{
Research Paper: \\ Toxicity and Antiparasitic Efficacy of Essential Oils: Analyses of the Biochemical Compositions and Potencies
}

\author{
Emad Soleimani $^{1}$ (D, Hossein Sarmadian² (D, Mohammad Arjomand Zadegan ${ }^{3}$ (D), Reza Ghasemikhah ${ }^{4 *}$ (D), Hasan Taher Ahmadi ${ }^{5}$ (D) \\ 1. Student Research Committee, Arak University of Medical Sciences, Arak, Iran. \\ 2. Department of Infectious Disease, School of Medicine, Arak University of Medical Sciences. Arak, Iran. \\ 3. Department of Microbiology, Infectious Diseases Research Center (IDRC), School of Medicine, Arak University of Medical Sciences, Arak, Iran. \\ 4. Department of Pediatrics, School of Medicine, Arak University of Medical Sciences, Arak, Iran. \\ 5. Department of Parasitology and Mycology, School of Medicine, Arak University of Medical Sciences, Arak, Iran.
}

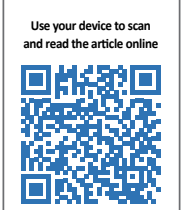

How to cite this paper Soleimani E, Sarmadian H, Arjomand Zadegan M, Ghasemikhah R, Taher Ahmadi H. Toxicity and Antiparasitic Efficacy of Essential Oils: Analyses of the Biochemical Compositions and Potencies. Iranian Journal of Toxicology. 2021; 15(1):1-8. http://dx.doi.org/10.32598/ijt.15.1.727.1

doi http://dx.doi.org/10.32598/ijt.15.1.727.1

\section{(c) (1) (\$)}

Article info:

Received: 11 Jul 2020

Accepted: 15 Sep 2020

Online Published: 01 Jan 2021

* Corresponding author:

Reza Ghasemikhah, PhD.

Address: Department of Pediatrics, School of Medicine, Arak University of Medical Sciences, Arak, Iran.

E-mail: ghasemikhah@yahoo.com

\section{ABSTRACT}

Background: Hydatidosis is a common disease of both humans and animals, resulting from infection with the larvae of Echinococcus granulosus tapeworm. The aim of this study was to investigate the antiparasitic (protoscolicidal) activities of three essential oils in vitro.

Methods: This study was designed to evaluate the biochemical composition and in vitro antiparasitic effects of Zataria multiflora, Origanum vulgare and Mentha pulegium essential oils. Gas chromatography was performed to identify the main components of the herbal oils. To determine the antiparasitic properties of the essential oils, live protoscoleces from hydatid cysts were exposed to three concentrations of the herbal oils and were incubated at $37^{\circ} \mathrm{C}$ for 5 , $10,15,20$ or 25 minutes.

Results: The biochemical analysis of these oils indicated that carvacrol and thymol were the major compounds of the Zataria oil. Further, carvacrol and thymol in Origanum essential oil and pulegone and piperitone in Mentha oil were the major compounds. The quickest and slowest antiparasitic effect was achieved from Zataria and Origanum (10\%) or from Zataria $(0.6 \%)$, respectively. The statistical analysis showed a significant difference between the mortality rate of protoscoleces exposed to $0.6 \%$ and $1 \%$ concentrations, respectively, of Zataria and Origanum at the predetermined exposure times $(\mathrm{P}<0.05)$. The three concentrations of Mentha had the same significant statistical differences $(\mathrm{P}<0.05)$.

Conclusion: Essential oils, Zataria multiflora, Origanum vulgare and Mentha pulegium had significant protoscolicidal activities that were dependent on the concentration of the oils and the exposure times.

Keywords: Protoscoleces, Essential oils, Zataria multiflora, Origanum vulgare, Mentha pulegium

\section{Introduction}

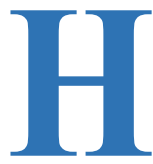

ydatid disease (hydatidosis) is caused by infection with Echinococcus larvae. The disease is severe and the treatment is complicated. The current treatment choice is surgery, which has serious side effects and is costly. The most complications of hydatid surgery are rupture of the cyst and recurrence of disease due to formation of secondary cysts. So far, various mechanical methods and chemical agents have been tested in order to prevent the formation of secondary hydatidosis. The antiparasitic ef- 
fects of direct electric current [1], magnetic field [2], xray [3], UV light [4], gamma irradiation [5], ultrasound $[6,7]$, nanoparticles [8], hypotonic saline [9], silver nitrate [10], vinegar [11] and various herbal extracts or essential oils have been evaluated on protoscoleces to certain extents [12-14].

Zataria multiflora, Origanum vulgare and Mentha pulegium have been widely used in traditional medicine, from which numerous therapeutic properties have been suggested. The most effective components of the Zataria multiflora, Origanum vulgare are thymal and caracrol, which are confirmed antibacterial, antifungal, and antiparasitic agents in vitro $[15,16]$. The essential oil from Mentha pulegium has been traditionally used as an antiseptic and antibacterial agent [13].

The current study aimed at evaluating the efficacy of micro and macro-emulsions of Zataria multiflora, Origanum vulgare and Mentha pulegium on protoscoleces of hydatid cyst origins.

\section{Materials and Methods}

Preparation of protoscoleces: This in vitro study was conducted on protoscoleces derived from hydatid cysts. Sheep hydatid cysts were collected from a slaughter house in Arak, Iran, and were transferred to the Parasitology Laboratories at Arak University of Medical Sciences. The contents of the cysts were completely evacuated by sterile syringes and washed 3 times with normal saline. The concentration of protoscoleces was set by normal saline so that the number of protoscoleces per $\mathrm{mL}$ of the suspension was 6000 at more than $90 \%$ viability. The suspension of live protoscoleces was transferred to a dark container and stored at $4^{\circ} \mathrm{C}$ for the subsequent experiments.

Viability test: The viability of protoscoleces was tested by $0.1 \%$ eosin staining. For this purpose 0.1 gram of eosin was dissolved in $100 \mathrm{~mL}$ of distilled water. Equal volumes of protoscoleces suspension and $0.1 \%$ eosin solution were mixed. After three minutes, the protoscoleces were examined under light microscopy. The stained protoscoleces were considered dead and the unstained ones were recorded as being alive [17].

Preparation of medicinal plants and essential oils: Zataria multiflora, Origanum vulgare and Mentha pulegium were obtained from the Agricultural Research Center in Isfahan province and were transferred to the laboratory of Infectious Diseases Research Center at the School of Medicine, Arak University of Medical Sci- ences. The young leaves form each plant were separated, dried in the dark at low humidity and used for the extraction of the essential oils. The oils from Zataria multiflora, Origanum vulgare and Mentha pulegium were prepared by steam distillation in a Clevenger apparatus. Briefly, a $30 \mathrm{~g}$ sample of the dried leaves was added to a $1000 \mathrm{~mL}$ distillation balloon to which $300 \mathrm{~mL}$ distilled water was added. The Clevenger's refrigerant and rotator of cold water were placed on the distillation balloon then the heating unit was turned on.

Micro emulsion preparation: The essential oils dissolved in Tween 80 (Polysorbate 80) were separated from the water on a magnet stirrer for approximately five minutes. Using this method, a milky appearing macroemulsion was prepared for each plant at $10 \%$ concentration. The bright appearing micro-emulsion was prepared at $0.6 \%$ or $1 \%$ dilution.

\section{Gas chromatography \& mass spectrometry}

Analysis of essential oils: The analysis of the oil samples was carried out, using an Agilent 7890 Ampere with a HP-5MS column $(30 \mathrm{~m} \times 0.25 \mathrm{~mm}$, film thickness $0.25 \mathrm{~mm}$ ). The column temperature was maintained at $50^{\circ} \mathrm{C}$ for $3 \mathrm{~min}$ and increased progressively to $300^{\circ} \mathrm{C}$ at a rate of $5^{\circ} \mathrm{C}$ per min, and kept constant at $300^{\circ} \mathrm{C}$ for $5 \mathrm{~min}$. The injector and interface temperatures were set at $270^{\circ} \mathrm{C}$ and $300^{\circ} \mathrm{C}$, respectively. The atomic mass unit ranged between $50^{\circ} \mathrm{C}$ and $300^{\circ} \mathrm{C}$. The flow rate of helium as the carrier gas was $2 \mathrm{~mL} / \mathrm{min}$. The oven was set at the same temperature as mentioned above for the gas chromatography. See Tables 1, 2 and 3.

Identification of components: The oils' components were identified and confirmed in comparison with their mass spectra and those of a computer library or with the authentic compounds.

Experiments: The experiments were conducted in eleven groups. Each group included six test tubes, each of which was used for a single incubation time. All of the test tubes contained $300 \mu \mathrm{L}$ protoscoleces suspension ( $\sim 1800$ protoscoleces) plus $300 \mu \mathrm{L}$ of the herbal materials. The tests were performed as follows:

1: $0.6,1$ or $10 \%$ concentration of Zataria multiflora was added to the first, second or third groups of test tubes, respectively.

2: $0.6,1$ or $10 \%$ concentration of Origanum vulgare was added to the fourth, fifth or sixth groups of test tubes, respectively. 


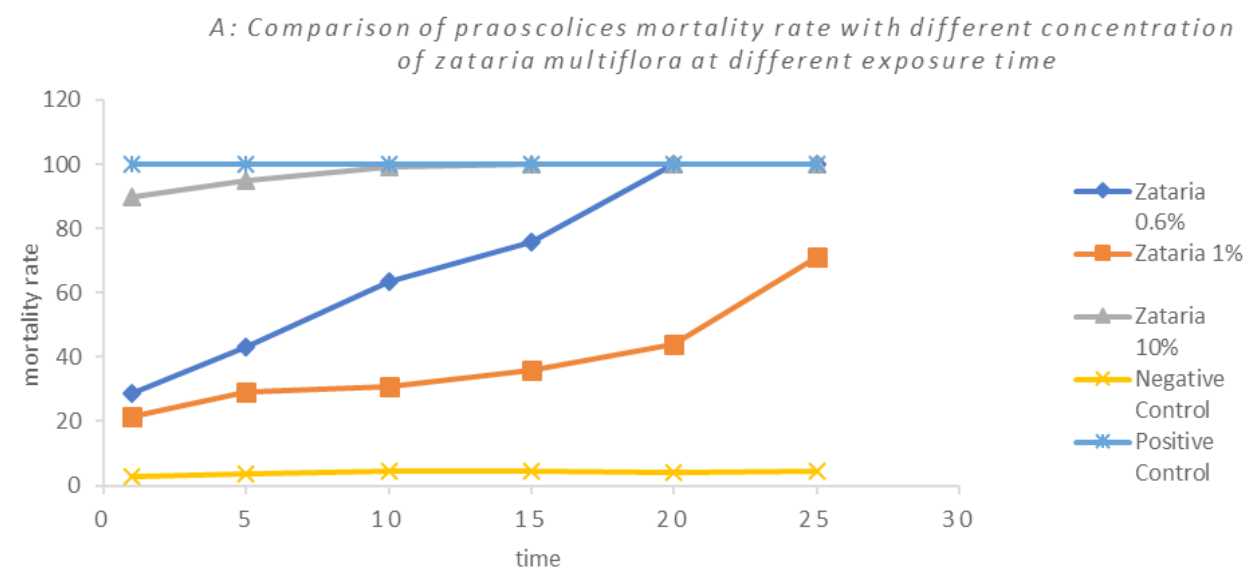

Figure 1. Comparison of protoscoleces mortality rates at varying concentrations of Zataria multiflora versus exposure times.

3: $0.6,1$ or $10 \%$ concentration of Mentha pulegium was added to the seventh, eighth or ninth groups of test tubes, respectively.

An aliquot of normal saline (negative control) or $20 \%$ concentration of hypertonic saline (positive control) was added to the tenth or eleventh group, respectively. The test tubes' contents were vortexed then incubated at $37^{\circ} \mathrm{C}$ for $1,5,10,15,20$ or $25 \mathrm{~min}$. Each experiment was repeated three times. At the end of each incubation time, the tubes contents were mixed with $0.1 \%$ eosin. The protoscoleces' mortality rate was examined and the numbers recorded under light microscopy.

Statistical analyses: Statistical analyses were performed on SPSS V. 16 for Windows. The data were presented as the mean values of three separate experiments and expressed as means \pm standard deviations for each group. The differences among the groups and the control were statistically analyzed by one-way ANOVA test. The statistical significance was defined as $\mathrm{P}<0.05$.

\section{Results}

Analyses of essential oils: The major compounds in the essential oils derived from the three plants were as follows: Carvacrol and thymol were found in Zataria and oregano while pulegone and piperitone were identified in Mentha.

Antiparasitic effects: The most potent antiparasitic effect was recorded in both Zataria and Origanum at 10\% concentration, which killed all protoscoleces after one minute exposure. The slowest antiparasitic effect was detected for Zataria at $0.6 \%$ concentration, which caused the death of all of the protoscoleces after 20 minutes of

Table 1. The essential oil composition of Mentha pulegium

\begin{tabular}{|c|c|c|c|}
\hline Peak No. & Retention Time (Min.) & Compound & Combined (\%) \\
\hline 1 & 4.199 & Octane & 1.479 \\
\hline 3 & 11.48 & 1,8-Cineole & 5.937 \\
\hline 5 & 15.578 & Borneol L & 1.547 \\
\hline 6 & 16.244 & Alpha Terpineol & 1.437 \\
\hline 7 & 17.601 & Pulegone & 6.842 \\
\hline 8 & 20.577 & Piperitenone & 30.120 \\
\hline 9 & 21.234 & Piperitenone Oxide & 18.307 \\
\hline 10 & 22.155 & Caryophyllene & 1.720 \\
\hline 11 & 25.714 & Caryophyllene Oxide & 2.597 \\
\hline
\end{tabular}


Table 2. Essential oil composition of Origanum vulgare

\begin{tabular}{cccc}
\hline Peak No. & Retention Time (Min.) & Compound & Combine (\%) \\
\hline 1 & 4.193 & Octane & 1.420 \\
\hline 3 & 8.207 & Alpha-Pinene & 1.789 \\
\hline 4 & 10.087 & Beta-Myrcene & 2.907 \\
\hline 5 & 10.961 & Alpha-Terpinene & 11.074 \\
7 & 11.284 & O-Cymene & 17.950 \\
\hline 8 & 12.475 & Gamma-Terpinene & 1.286 \\
10 & 15.838 & $4-T e r p i n e o l$ & 49.427 \\
\hline
\end{tabular}

Table 3. Essential oil composition of Zataria multiflora

\begin{tabular}{|c|c|c|c|}
\hline Peak No. & Retention Time (Min.) & Compound & Combine (\%) \\
\hline 1 & 4.188 & Octane & 3.141 \\
\hline 2 & 8.22 & Alpha-pinene & 4.699 \\
\hline 3 & 10.066 & 2-ethyl-Butanal & 1.063 \\
\hline 4 & 10.373 & Decane & 1.500 \\
\hline 5 & 10.94 & (+)-2-Carene & 1.883 \\
\hline 6 & 11.273 & Cymol & 14.372 \\
\hline 7 & 12.317 & Gamma-terpinene & 6.006 \\
\hline 8 & 13.529 & Linalool & 1.116 \\
\hline 9 & 16.341 & Dodecane & 1.506 \\
\hline 10 & 17.284 & Thymyl metthyl ether & 2.438 \\
\hline 11 & 19.227 & Thymol & 33.894 \\
\hline 12 & 19.465 & Carvacrol & 7.759 \\
\hline 13 & 20.387 & AcetateThymol & 2.024 \\
\hline 14 & 22.092 & Trans (beta)-Caryophyllene & 3.620 \\
\hline 15 & 22.515 & Aromadendrene & 1.854 \\
\hline 16 & 23.723 & Ledene & 1.142 \\
\hline 17 & 25.555 & (+) Spathulenol & 1.924 \\
\hline 18 & 25.666 & Caryophyllene oxide & 1.242 \\
\hline
\end{tabular}




\section{B: Comparison of praoscolices mortality rate \\ with different concentration of organum \\ vulgare at different exposure time}

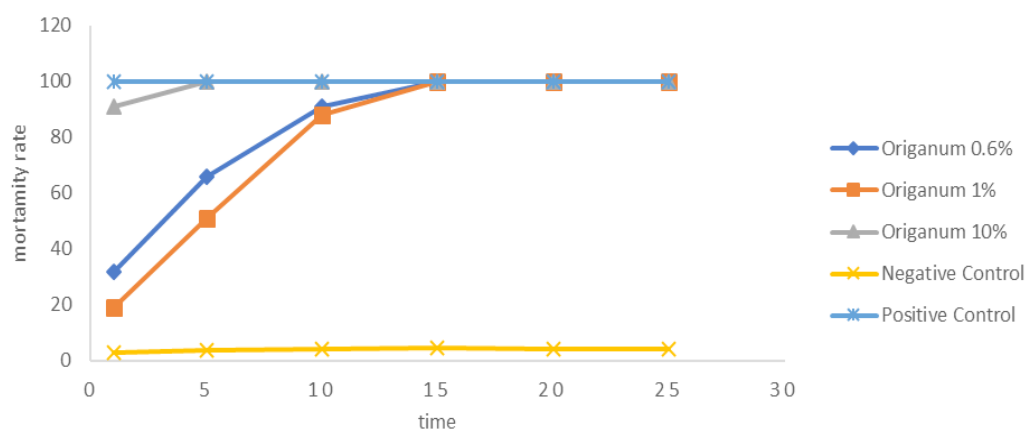

Figure 2. Comparison of protoscoleces mortality rates at varying concentrations of Organum vulgare versus exposure times

exposure (Table 1). The survival rates of the protoscoleces from each herbal material are shown in Figure 1.

The statistical analysis revealed a significant difference between the mortality rate of protoscoleces exposed to Zataria and Origanum at $0.6 \%$ and $1 \%$ concentrations, respectively, versus the exposure times $(\mathrm{P}<0.05)$. However, the three Mentha oils concentrations revealed similar statistical differences $(\mathrm{P}<0.05)$. See details in Figures 1, 2 and 3.

\section{Discussion}

Hydatidosis is a chronic disease that may occur in various parts of the world. The application of benzimidazole and the Punctuation, Aspiration, Injection and Re-aspiration (PAIR) approach to the management of this disease dates back to many decades ago, even though a definitive medical cure is out of reach $[18,19]$. Except for surgery, that is the popular approach, there is no consensus on the best conservative treatment for hydatidosis within the medical community $[18,20,21]$. The most common risk of surgery is the rupture of cysts, which may lead to the spread of protoscoleces and formation of secondary cysts in the patient at a minimum $[11,22]$. The World Health Organization has recommended using herbal medicines and natural alternatives against parasitic diseases [23].

For this reason, plant-derived agents have attracted the attention of many researchers and clinicians, because most herbal compounds are safe and some of them are consumed as foods $[15,17,24]$.

In previous studies, the effect of several herbal extracts and their essential oils were tested on protoscoleces from hydatid cysts $[16,17,25]$. In the present study, the antiparasitic effects of three medicinal herbs at varying concentrations were tested versus their exposure times (Figures 1, 2 and 3). Comparing the lethal dosages on the hydatid protoscoleces revealed that the effect of the macro-emulsion was more potent than that of the microemulsion. However, the micro-emulsion at low concentration provided desirable effects.

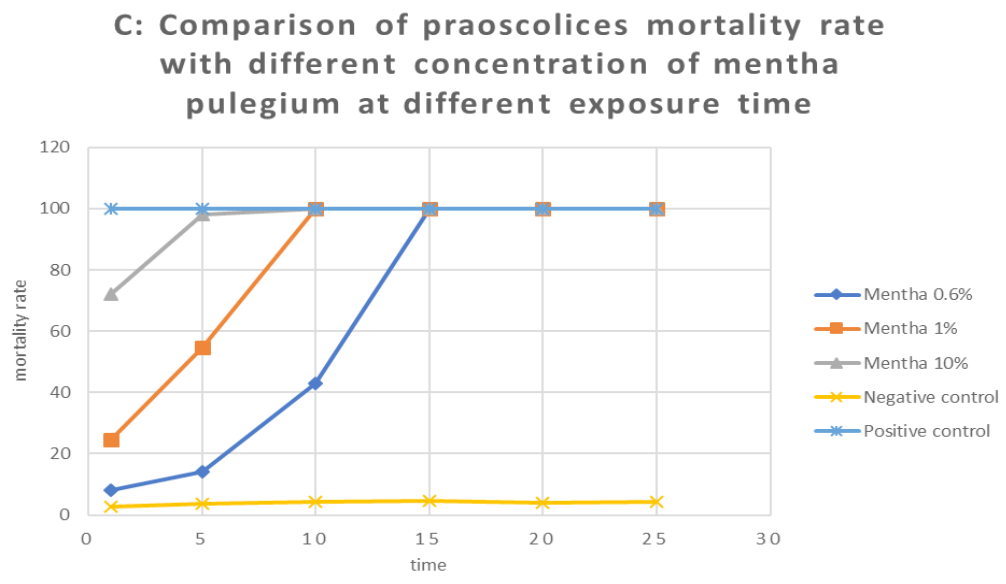

Figure 3. Comparison of protoscoleces mortality rates at varying concentrations of Menthe pulegium versus exposure times 
Studies have investigated the effects of various plants' essential oils on protoscoleces. For examples, the essential oils from Satureja khuzistanica and Nigella sativa have destroyed $100 \%$ of protoscoleces in 10 minutes. Also, Rhus coriaria has been shown to kill $100 \%$ of protoscoleces over a 30 -min exposure $[17,26]$. In this context, the essential oils we prepared were more potent than those used in previous studies. For instance, the essential oil from Zataria multiflora and Origanum vulgare killed $100 \%$ of protoscoleces in one minute while the oil extracted from Mentha pulegium achieved the same effect in 10 minutes. The differences in the antiparasitic effects of the essential oils prepared in the current study might be due to differences in their solvents.

In previous studies, DMSO was used as the solvent of essential oils; however, the solubility in water is not comparable to that of DMSO [27], which may induce agglomeration. Of note, the essential oils prepared in this study in macro- and micro-emulsion forms were entrapped in nano-scale vesicles, which made them more potent than other forms of essential oils.

The antiparasitic effects of Zataria multiflora, Origanum vulgare and Mentha pulegium depended on the concentrations of their essential oil and exposure time. In this respect, the Origanum's essential oil at $0.6 \%$ concentration demonstrated the best antiparasitic effect compared to those extracted from Zataria and Mentha plants.

\section{Conclusions}

Based on the findings of this study, the extraction, purification and application of natural compounds, such as essential oils, from medicinal plants are promising steps to developing effective treatment for hydatid cysts in humans. We discovered that the essential oil derived from Origanum at $0.6 \%$ concentration provided the best antiparasitic effect compared to those from Zataria and Mentha plants. The conservative approach suggested by this study is likely to provide an equally effective treatment for parasitic cysts while offering a safer approach than surgery. Limitations of the Study: Obtaining non-infectious hydatid cysts with $90 \%$ viable protoscolices was one of the limitations of this study.

Recommendations for Future Research: For better effects of the essential oils on hydatid cysts protoscoleces, we recommend that the optimal oil concentrations be determined on protoscoleces grown in a cell culture medium, such as RPMI.

\section{Ethical Considerations}

\section{Compliance with ethical guidelines}

All instructions and ethical considerations were followed in this project as set by the Research Council of Arak University of Medical Science. The Ethics Committee ethically approved this study (Code: IR.ARAKMU. REC.1394.310).

\section{Funding}

This paper was extracted from the master thesis of the first author, Student Research Committee, Arak University of Medical Sciences, Arak (Grant no. 1117).

\section{Author's contributions}

All authors contributed in preparing this article.

\section{Conflict of interest}

The authors declared no conflict of interests.

\section{Acknowledgements}

The authors express their gratitude to the Research Council, Deputy of Education, Arak University of Medical Sciences for funding this research project.

\section{References}

[1] Dalimi A, Ghasemikhah R, Hashemi Malayeri B. Echinococcus granulosus: Lethal effect of low voltage direct electric current on hydatid cyst protoscoleces. Exp Parasitol. 2005; 109(4):237-40. [DOI:10.1016/j.exppara.2004.12.014] [PMID]

[2] Sarmadian H, Ghasemikhah R, Mirmoradi F. The toxic effect of magnetic field on protoscoleces of hydatid cyst in vitro. Iran J Toxicol. 2013; 7(22):926-31. http://ijt.arakmu.ac.ir/ article-1-257-en.htm

[3] Bao YX, Mao R, Qi HZ, Zhang YF, Ni YQ, Xie ZR, et al. X-ray irradiation against Echinococcus multilocularis protoscoleces in vitro. Zhongguo Ji Sheng Chong Xue Yu Ji Sheng Chong Bing Za Zhi. 2011; 29(3):208-11. [PMID]

[4] Zhao Y, Gui W, Zhang Y, Mo G, Li D, Chong S. Inhibitory effect of ionizing radiation on echinococcus granulosus hydatid cyst. Diseases. 2019; 7(1):23. [DOI:10.3390/diseases7010023] [PMID] [PMCID]

[5] Alam-Eldin YH, Badawy AF. Destructive effect of gamma irradiation on Echinococcus granulosus metacestodes. Parasitol Res 2015; 114(8):3145-50. [DOI:10.1007/s00436-015-4533-9] [PMID] 
[6] Zhang J, Ye B, Kong J, Cai H, Zhao Y, Han X, et al. In vitro protoscolicidal effects of high-intensity focused ultrasound enhanced by a superabsorbent polymer. Parasitol Res. 2013; 112(1):385-91. [DOI:10.1007/s00436-012-3176-3] [PMID]

[7] Zou X, Wang J, Zhao H, Zhang J, Wu W, Ye B. Echinococcus granulosus: Protoscolicidal effect of high intensity focused ultrasound. Exp Parasitol. 2009; 121(4):312-6. [DOI:10.1016/j. exppara.2008.12.002] [PMID]

[8] Lashkarizadeh MR, Asgaripour K, Saedi Dezaki E, Fasihi Harandi M. Comparison of scolicidal effects of amphotricin b, silver nanoparticles, and foeniculum vulgare mill on hydatid cysts protoscoleces. Iran J parasitol. 2015; 10(2):206-12. [PMID] [PMCID]

[9] Kayaalp C, Balkan M, Aydin C, Ozgurtas T, Tanyuksel M, Kirimlioglu, V, et al. Hypertonic saline in hydatid disease. World J Surg. 2001; 25(8):975-9. [DOI:10.1007/s00268-0010065-9] [PMID]

[10] Hosseini S, Ghanbarzadeh K, Barzin J, Sadjjadi SM, Tanideh N, Mehrabani D. In vitro protoscolicidal effects of hypertonic glucose on protoscolices of hydatid cyst. Korean J Parasitol. 2006; 44(3):239-42. [DOI:10.3347/kjp.2006.44.3.239] [PMID] [PMCID]

[11] Hajihossein R, Eslamirad Z, Mosayebi M, Ghasemikhah $R$, Didehdar M. In vitro effects of vinegar on protoscolices of hydatid cyst. Asian Pac J Trop Dis. 2015; 5(3):210-3. [DOI:10.1016/S2222-1808(14)60655-1]

[12] Elissondo MC, Pensel PE, Denegri GM. Could thymol have effectiveness on scolices and germinal layer of hydatid cysts? Acta Tropica. 2013; 125(3):251-7. [DOI:10.1016/j.actatropica.2012.12.007] [PMID]

[13] Maggiore MA, Albanese AA, Gende LB, Eguaras MJ Denegri JM, Elissondo MC. Anthelmintic effect of Mentha spp. essential oils on Echinococcus granulosus protoscoleces and metacestodes. Exp Parasitol. 2012; 110(3):1103-12. [DOI:10.1007/s00436-011-2595-x] [PMID]

[14] Moazeni M, Roozitalab A. High scolicidal effect of Zataria multiflora on protoccoleces of hydatid cyst:an in vitro study. Comp Clin Path. 2012; 21(1):99-104. [DOI:10.1007/s00580-010-1069-3]

[15] Khalili M, Vahidi A. The anti-microbial effect of Zataia multiflora drops on three bacterial species cause gastrointestinal disease. World J Med Sci. 2006; 1(2):162-3. https:/ /www. idosi.org/wjms/1(2)2006/23.pdf

[16] Pensel PE, Maggiore MA, Gende LB, Eguaras MJ, Denegri MG, Elissondo MC. Efficacy of essential oils of thymus vulgaris and origanum vulgare on echinococcus granulosus. Interdiscip Perspect Infect Dis. 2014; 2014:693289. [DOI:10.1155/2014/693289] [PMID] [PMCID]

[17] Mahmoudvand H, Ayatollahi Mousavi SA, Sepahvand A, Sharififar F, Ezatpour B, Gorohi f, et al. Protoscolecidal effect of berberis vulgaris root extract and its main compound, berberine in cystic echinococcosis. Iran J Parasitol. 2014; 9(4):503-10. [DOI:10.1155/2014/602436] [PMID] [PMCID]

[18] Tamarozzi F, Vuitton L, Brunetti E, Vuitton DA, Koch S Non-surgical and non-chemical attempts to treat echinococcosis: Do they work? Parasite. 2014; 21:75. [DOI:10.1051/parasite/2014071] [PMID] [PMCID]

[19] Maryam Karimi M, Ghasemikhah R, Mirahmadi H, Spotin A, Rouhani S, Seyyed Tabaei SJ. Discrimination of mixed infections of echinococcus species based on in silico sequence analysis: A new way of reflecting overlapped strains in indigenous areas. Arch Clin Infect Dis. 2017; 12(4):e14168. [DOI:10.5812/archcid.14168]

[20] Abedi B, Maghsood AH, Khansarinejad B, Fallah M, Matini M, Gholami S, et al. Genotyping of Echinococcus granulosus isolates from livestock based on mitochondrial cox1 gene, in the Markazi province, Iran. J Parasit Dis. 2019; 43(4):592-6. [DOI:10.1007/s12639-019-01132-4] [PMID] [PMCID]

[21] Fathi S, Ghasemikhah R, Mohammadi R, Tohidi F, Sharbatkhori M. Seroprevalence of hydatidosis in people referring to reference laboratory of Gorgan, Golestan province, Northern Iran 2017. Iran J Parasitol. 2019; 14(3):436-43. [DOI:10.18502/ ijpa.v14i3.1483] [PMID] [PMCID]

[22] Ghasemikhah R, Shahdoust M, Sarmadian H, Rezaei M, Ghorbanzadeh B, Gorji A, et al. Echinococcosis in livestock slaughtered in Arak industrial abattoir in Central Iran during 2006 to 2012. West Indian Med J. 2015. [DOI:10.7727/ wimj.2015.153]

[23] Tiuman TS, Ueda-Nakamura T, Cortez DAG, Filho BPD, Morgado-Díaz JA, de Souza W, et al. Antileishmanial activity of parthenolide, a sesquiterpene lactone isolated from tanacetum parthenium. Antimicrob Agents Chemother. 2005; 49(1):176-82. [DOI:10.1128/AAC.49.11.176-182.2005] [PMID] [PMCID]

[24] Ghasemikhah R, Tabatabaiefar MA, Shariatzadeh SA, Shahbazi A, Hazratian T. A PCR-based molecular detection of strongyloides stercoralisin human stool samples from $\mathrm{Ta}$ briz City, Iran. Sci Pharm. 2017; 85(17):3-6. [DOI:10.3390/scipharm85020017] [PMID] [PMCID]

[25] Moazeni M, Larki S, Pirmoradi G, Rahdar M. Scolicidal effect of the aromatic water of Zataria multiflora: An in vitro study. Comp Clin Path. 2015; 24(5):1057-62. [DOI:10.1007/ s00580-014-2030-7]

[26] Moazeni M, Mohseni M. Sumac ((Rhus coriaria L.): Scolicidal activity on hydatid cyst protoscolices. Surg Sci. 2012; 3(9):452-6. [DOI:10.4236/ss.2012.39089]

[27] Hili P, Evans CS, Veness RG. Antimicrobial action of essential oils: The effect of dimethylsulphoxide on the activity of cinnamon oil. Lett Appl Microbiol. 1997; 24(4):269-75. [DOI:10.1046/j.1472-765X.1997.00073.x] [PMID] 
This Page Intentionally Left Blank 